\title{
Searching for Relational Patterns in Data
}

\author{
Sinh Hoa Nguyen and Andrzej Skowron \\ Institute Mathematics, Warsaw University \\ Warsaw, 02-097, Banacha Str. 2 \\ emails: \{hoa,skowron\}@mimuw.edu.pl
}

\begin{abstract}
We consider several basic classes of tolerance relations among objects. These (global) relations are defined from some predefined similarity measures on values of attributes. A tolerance relation in a given class of tolerance relations is optimal with respect to a given decision table $\mathbf{A}$ if it contains only pairs of objects with the same decision and the number of such pairs contained in the relation is maximal among all relations from the class. We present a method for (sub-)optimal tolerance relation learning from data (decision table). The presented method is based on rough set approach. We show that for some basic families of tolerance relations this problem can be transformed to a relative geometrical problem in a real affine space. Hence geometrical computations are becoming useful tools for solving the problem of global tolerance relation construction. The complexity of considered problems can be evaluated by the complexity of the corresponding geometrical problems. We propose some efficient heuristics searching for an approximation of optimal tolerance relations in considered families of tolerance relations. The global tolerance relations can be treated as patterns in the cartesian product of the object set.We show how to apply the relational patterns (global tolerance relations) in clustering and classification of objects.
\end{abstract}

\section{Introduction}

In rough set theory [10] the notion of set approximation has been introduced by using equivalence relation defined on the set of objects. In some cases, it is necessary to generalize this notion by using tolerance relation (similarity relation) $[4,15]$. The tolerance relation can be defined in many different ways. Often tolerance relations are given by introducing some local similarity measures on values of attributes together with some rules of composing those local similarities into global ones.

One of the main problem of methodology for data mining is to develop methods for automatic pattern extraction from data. In our previous papers [7, 8] we have suggested to search for such patterns in the form of templates. Using them it was possible to decompose a given table into a family of subtables corresponding to these patterns and to create subdomains of a given space of objects. The objects from any subdomain have many common features what suggests that they can create a "regular" subdomain for which strong decision rules can be generated. 
In this paper we consider patterns defined by tolerance relations. These patterns correspond to some (sub-)optimal tolerance relations extracted from data. In this way we propose rather to search for (sub-)optimal tolerance relations from data in predefined classes of tolerance relations than by assuming apriori their form (as it is often done when clustering methods are used).

In searching for tolerance relations from data we follow a method proposed in [14] based on rough sets. We propose a method of searching for (sub-)optimal tolerance relation (with respect to the number of the pairs of objects with the same decision from this relation) by transforming the considered problem to the problem of approximate description of some regions in affine space $R^{k}$, where $k$ is equal to the number of (conditional) attributes.

We consider several classes of tolerance relation. Any class is characterized by a first order formula and some parameters which are tuned in the optimization process. For any of these classes we propose strategies searching for (sub-)optimal tolerance relation in it i.e. described by a maximal set of object pairs having the same decision. We illustrate how the extracted patterns can be used for cluster construction and classification of new objects.

\section{Basic notions}

\subsection{Rough set preliminaries}

An information system is defined by a pair $\mathbf{A}=(U, A)$, where $U$ is a non-empty, finite set of objects called universe, $A=\left\{a_{1}, \ldots, a_{k}\right\}$ is a non-empty, finite set of attributes, i.e. $a_{i}: U \rightarrow V_{a_{i}}$ for $i \in\{1, \ldots, k\}$, where $V_{a_{i}}$ is called the domain of the attribute $a_{i}$.

The information space of $A$ is defined by $I N F_{A}=\prod_{a \in A} V_{a}$. We define the information function $I n f_{A}: U \rightarrow I N F_{A}$ by

$$
\operatorname{In} f_{A}(x)=\left(a_{1}(x), \ldots, a_{k}(x)\right), \text { for any } x \in U .
$$

Any object $x \in U$ is represented by its information vector $\operatorname{In} f_{A}(x)$.

A decision table $\mathbf{A}=(U, A \cup\{d\})$, where $d \notin A$ is a distinguished attribute called decision is a special case of information systems.

For any information system $\mathbf{A}=(U, A)$ and a subset $B \subseteq A$ the $B$-indiscernibility relation $I N D_{B}$ can be defined by

$$
x I N D_{B} y \Leftrightarrow \forall_{a \in B}[a(x)=a(y)]
$$

Obviously, $I N D_{B}$ is an equivalence relation. We denote by $[x]_{I N D_{B}}=\{y$ : $\left.\langle x, y\rangle \in I N D_{B}\right\}$ the equivalence class defined by the object $x \in U$.

For any $Y \subseteq U$ one can define the lower approximation and the upper approximation of $X$ by

$$
\underline{X}=\left\{x \in U:[x]_{I N D_{B}} \subseteq X\right\} ; \bar{X}=\left\{x \in U:[x]_{I N D_{B}} \bigcap Y \neq \emptyset\right\} \text {, respectively. }
$$

The pair $(\underline{X}, \bar{X})$ is referred to as the rough sets of $X$. 


\subsection{Tolerance relation}

Indiscernibility relation is a useful tool of rough set theory, but in many cases it is not sufficient, in particular, when we deal with real value attributes. In this case almost every object differs from another. The equivalence classes divide universe into tiny classes. Therefore the description of a subset of $U$ is complicated (with respect to the number of equivalence classes) and not enough general. The standard rough set model can be generalized by assuming any type of binary relation (on attribute values) instead of the equivalence relation.

In this paper we consider a family of relations $\tau \subseteq U \times U$ which are reflexive (i.e. $\forall x \in U\langle x, x\rangle \in \tau)$ and symmetric (i.e. $\forall_{x, y \in U}(\langle x, y\rangle \in \tau \Rightarrow\langle y, x\rangle \in \tau)$ ). Such relations are called the tolerance relations.

For any $x \in U$ the tolerance class $[x]_{\tau}$ can be defined by

$$
[x]_{\tau}=\{y \in U:\langle x, y\rangle \in \tau\}
$$

We say, that the tolerance relation $\tau$ identifies objects $x$ and $y$ if $\langle x, y\rangle \in \tau$; otherwise we say that it discerns them.

One can define the lower approximation and the upper approximation of any subset $X \subseteq U$ by

$$
\underline{\tau(X)}=\left\{x \in U:[x]_{\tau} \subseteq X\right\} ; \overline{\tau(X)}=\left\{x \in U:[x]_{\tau} \cap X \neq \emptyset\right\} \text {, respectively. }
$$

Let $\mathbf{A}=(A, U \cup\{d\})$. be a decision table. A similarity measure for an attribute $a \in A$ is a positive function $\delta_{a}: U \times U \rightarrow \Re^{+} \cup\{0\}$ satisfing the following conditions:

1. $\delta_{a}(x, x)=0$

2. $\delta_{a}(x, y)=\delta_{a}(y, x)$;

Having a family of similarity measures $\left\{\delta_{a_{i}}\right\}_{a_{2} \in A}$ the tolerance relation $\tau$ $\subseteq U \times U$ can be defined by

$$
\langle x, y\rangle \in \tau \Leftrightarrow \Psi_{R}\left(\delta_{a_{1}}(x, y), \delta_{a_{2}}(x, y), \ldots, \delta_{a_{k}}(x, y)\right)=\text { true }
$$

where $\Psi\left(\xi_{1}, \xi_{2}, \ldots, \xi_{k}\right)$ is a first order logic propositional formula and $\Psi_{R}$ is its realization in a relational structure of real numbers such that $\Psi_{R}(0, \ldots, 0)=$ true.

By $C_{k}$ we denote the set $\left\{\left(r_{1}, r_{2}, \ldots, r_{k}\right) \in R^{k}: 0 \leq r_{i}\right.$, for $\left.i=1, \ldots, k\right\}$. For any relation $\tau$ defined by (1), the interpretation of $\tau$ is defined by

$$
\bar{\tau}:=\left\{\left(r_{1}, r_{2}, \ldots, r_{k}\right) \in C_{k}: \Psi_{R}\left(r_{1}, r_{2}, \ldots, r_{k}\right)=\text { true }\right\} \subseteq C_{k} .
$$

One can define different tolerance relations using different formulas $\Psi\left(\xi_{1}, \ldots, \xi_{k}\right)$. We list some basic families of parameterized tolerance relations used in the paper:

1. $\langle x, y\rangle \in \tau\left(\varepsilon_{1}, \ldots, \varepsilon_{k}\right) \Leftrightarrow \forall_{a_{i} \in A}\left[\delta_{a_{i}}(x, y) \leq \varepsilon_{i}\right]$

2. $\langle x, y\rangle \in \tau\left(w_{1}, \ldots, w_{k}, w\right) \Leftrightarrow \sum_{a_{2} \in A} w_{i} \cdot \delta_{a_{i}}(x, y)+w \leq 0$

3. $\langle x, y\rangle \in \tau\left(w_{1}, \ldots, w_{k}, w\right) \Leftrightarrow \sum_{a_{1} \in A} w_{i} \cdot \delta_{a_{i}}^{2}(x, y)+w \leq 0$

4. $\langle x, y\rangle \in \tau\left(\varepsilon_{1}, \ldots, \varepsilon_{k}\right) \Leftrightarrow \exists_{a_{i} \in A}\left[\delta_{a_{i}}(x, y) \leq \varepsilon_{i}\right]$

5. $\langle x, y\rangle \in \tau(w) \Leftrightarrow \prod_{a_{2} \in A} \delta_{a_{i}}(x, y) \leq w$ 
where $\delta_{a_{i}}$ is a predefined similarity measure for $i=1, \ldots, k$ and $\varepsilon_{i}, \varepsilon, w_{i}, w$ are real numbers, called parameters.

A tolerance relation $\tau \subseteq U \times U$ is consistent with a decision table $\mathbf{A}=$ $(A, U \cup\{d\})$ if

$$
\langle x, y\rangle \in \tau \Rightarrow(d(x)=d(y)) \vee\left(\langle x, y\rangle \in I N D_{A}\right)
$$

for any objects $x, y \in U$.

The relation $\tau$ is optimal in the family $\mathcal{T}$ for a given $\mathbf{A}$ if $\tau$ contains the maximal number of pairs of objects among tolerance relations from $\mathcal{T}$ consistent with A.

\section{Extraction of global tolerance relation from data}

Let $\mathbf{A}=(A, U \cup\{d\})$ be a decision table and let $\delta_{a}$ be a similarity measure for any attribute $a \in A$. The problem of extracting a tolerance relation in a given class $\mathcal{T}$ is a searching problem for parameters such that the tolerance relation with the parameters found in searching process is optimal. The searching problem for the optimal tolerance relation is of high complexity.

Our goal is to search for a sub-optimal tolerance relation that discerns between all pairs of objects with different decisions and identifies maximal number of pairs of objects with the same decision.

In the first stage of tolerance relation construction, we define a new decision table $\mathbf{B}$ called the similarity table. The table $\mathbf{B}=\left(U^{\prime}, A^{\prime} \cup\{D\}\right)$ is defined assuming given $\mathbf{A}$ and the set of similarity measures $\left\{\delta_{a}\right\}_{a \in A}$ by

$$
U^{\prime}=U \times U ; A^{\prime}=\left\{\delta_{a}\right\}_{a \in A} ; \text { and } D(x, y)=\left\{\begin{array}{l}
0 \text { if } d(x)=d(y) \\
1 \text { otherwise }
\end{array}\right.
$$

The set of objects of the similarity table $\mathbf{B}$ (for a table $\mathbf{A}$ ) is equal to the set of all pairs of objects from table $\mathbf{A}$ and the attribute values are the values of the similarity measure functions for pairs of objects. The new table has a binary decision. The decision value for any pair of objects is equal to 0 if the objects have the same decision in the original table $\mathbf{A}$, and 1 otherwise.

The searching problem for a sub-optimal tolerance relation for table $\mathbf{A}$ among relations from a given class $\mathcal{T}$ of tolerance relations can be considered as the problem of decision rule extraction from the decision table $\mathbf{B}$. We are looking for decision rules describing the decision class corresponding to $D=0$, i.e. the class associated with pairs of objects of the table $\mathbf{A}$ with the same decision. Our goal is to search for the rule of the form $\Psi\left(a_{1}^{\prime}(u), a_{2}^{\prime}(u), \ldots, a_{k}^{\prime}(u)\right) \Rightarrow(D(u)=0)$ satisfied by as many as possible objects $u \in U^{\prime}$. 


\begin{tabular}{||l|l|l||l|l|l||}
\hline \hline Vit.A & Vit.C & Fruit & Vit.A & Vit.C & Fruit \\
\hline 1.0 & 0.6 & Apple & 2.0 & 0.7 & Pear \\
\hline 1.75 & 0.4 & Apple & 2.0 & 1.1 & Pear \\
\hline 1.3 & 0.1 & Apple & 1.9 & 0.95 & Pear \\
\hline 0.8 & 0.2 & Apple & 2.0 & 0.95 & Pear \\
\hline 1.1 & 0.7 & Apple & 2.3 & 1.2 & Pear \\
\hline 1.3 & 0.6 & Apple & 2.5 & 1.15 & Pear \\
\hline 0.9 & 0.5 & Apple & 2.7 & 1.0 & Pear \\
\hline 1.6 & 0.6 & Apple & 2.9 & 1.1 & Pear \\
\hline 1.4 & 0.15 & Apple & 2.8 & 0.9 & Pear \\
\hline 1.0 & 0.1 & Apple & 3.0 & 1.05 & Pear \\
\hline
\end{tabular}

Table 1. Apples and pears

\section{Geometrical interpretation}

In this section we show that some families of tolerance relations have clear geometrical interpretations, i.e. they can be described in a straightforward way as subsets of real affine space $R^{k}$. Therefore the searching problem for a sub-optimal tolerance relation can be reduced to searching for an approximate description of the corresponding subset of real affine space $R^{k}$.

For a decision table $\mathbf{A}=(U, A \cup\{d\})$ with $k$ conditional attributes and a set $\left\{\delta_{a}\right\}_{a \in A}$ of predefined similarity measures we build the similarity table $\mathbf{B}=\left(U^{\prime}, A^{\prime} \cup\{D\}\right)$. Every object $u \in U^{\prime}$ can be represented by a point $p(u)=$ $\left[a_{1}^{\prime}(u), \ldots, a_{k}^{\prime}(u)\right] \in R^{k}$ of one of two categories "white" or "black". A point $p(u) \in R^{k}$ is "white" iff $\left\{u^{\prime} \in U^{\prime}: p\left(u^{\prime}\right)=p(u)\right\}$ is non-empty and it consists of objects with the decision $D=0$ only; otherwise $p(u)$ is "black". Below we present a geometrical interpretations of some standard tolerance relations. As a similarity measures we take the functions: $\delta_{a}(x, y)=|a(x)-a(y)|$ for any attribute $a \in A$. We take as an example a table with two attributes representing the quantity of vitamin $\mathrm{A}$ and $\mathrm{C}$ in apples and pears.

We want to extract the similarities of fruits of one category. The data about apples and pears are shown in Figure 1.

Below we present a geometrical interpretations of some standard tolerance relations in the space of pairs of objects from the fruit table.

1. The relation, called the descriptor conjunction, is defined by

$$
\langle x, y\rangle \in \tau_{1}\left(\varepsilon_{1, \ldots,}, \varepsilon_{k}\right) \Leftrightarrow \bigwedge_{a_{i} \in A}\left[\delta_{a_{i}}(x, y) \leq \varepsilon_{i}\right]
$$

where $\varepsilon_{1}, \ldots, \varepsilon_{k} \in R^{+}$. The interpretation of $\tau_{1}\left(\varepsilon_{1, \ldots,} \varepsilon_{k}\right)$ (see (2)) is given by

$$
\overline{\tau_{1}\left(\varepsilon_{\left.1, \ldots, \varepsilon_{k}\right)}\right.}=\left\{\left(r_{1}, \ldots, r_{k}\right) \in C_{k}: 0 \leq r_{i} \leq \varepsilon_{i} \text { for } i=1, \ldots, k\right\}
$$


$\overline{\tau_{1}\left(\varepsilon_{1}, \ldots, \varepsilon_{k}\right)}$ is an interval in $R^{k}$ with boundaries $\varepsilon_{1}, \varepsilon_{2}, \ldots, \varepsilon_{k}$; it is attached to the origin $O$ of axes (Figure 2b). When $\varepsilon_{1}=\ldots=\varepsilon_{k}=\varepsilon$ instead of general interval in $R^{k}$ we have hypercubes (Figure 2a). By $\mathcal{T}_{1}$ we denote the family of all hypercubes and by $\mathcal{T}_{2}$ we denote the family of all relations defined by (3).

2. The linear combination relation is defined by

$$
\langle x, y\rangle \in \tau_{2}\left(w_{1, \ldots,} w_{k}, w\right) \Leftrightarrow \sum_{a_{i} \in A} w_{i} \cdot \delta_{a_{i}}(x, y)+w \leq 0
$$

where $w_{1}, \ldots, w_{k}, w \in R$. The interpretation of $\tau_{2}\left(w_{1}, \ldots, w_{k}, w\right)$ is given by

$$
\overline{\tau_{2}\left(w_{1}, \ldots, w_{k}, w\right)}=\left\{\left(r_{1}, \ldots, r_{k}\right) \in C_{k}: \sum_{i=1}^{k} w_{i} \cdot r_{i}+w \leq 0\right\}
$$

Hence $\overline{\tau_{2}\left(w_{1}, \ldots, w_{k}, w\right)}$ is a region below the hyperplane $\sum_{i=1}^{k} w_{i} \cdot x_{i}+w=0$ in $C_{k}$ (Figure $3 \mathrm{a}$ ). By $\mathcal{T}_{3}$ we denote the family of all tolerance relations of the form $\tau_{2}\left(w_{1, \ldots,}, w_{k}, w\right)$.

3. A linear combination can be extended to the higher order combination. We consider the relation defined by square combination of similarity measures

$$
\langle x, y\rangle \in \tau_{3}\left(w_{1}, \ldots, w_{k}, w\right) \Leftrightarrow \sum_{a_{i} \in A} w_{i} \cdot \delta_{a_{i}}^{2}(x, y)+w \leq 0 .
$$

where $w_{1}, \ldots, w_{k}, w \in R$. The interpretation of $\tau_{3}\left(w_{1}, \ldots, w_{k}, w\right)$ is given by

$$
\overline{\tau_{3}\left(w_{1}, \ldots, w_{k}, w\right)}=\left\{\left(r_{1}, \ldots, r_{k}\right) \in C_{k}: \sum_{i=1}^{k} w_{i} \cdot r_{i}^{2}+w \leq 0\right\}
$$

Hence $\overline{\tau_{3}\left(w_{1}, \ldots, w_{k}, w\right)}$ is a region in $C_{k}$ bounded by ellipsoid (Figure $3 \mathrm{~b}$ ). By $\mathcal{T}_{4}$ we denote the family of all tolerance relations of the form (4)

4. The next relation called "min" is defined by the formula

$$
\langle x, y\rangle \in \tau_{4}(\varepsilon) \Leftrightarrow \min _{a_{i} \in A}\left\{\delta_{a_{i}}(x, y)\right\} \leq \varepsilon,
$$

where $\varepsilon$ is a non-negative real. Hence $\overline{\tau_{4}(\varepsilon)}=\bigcup_{i=1}^{k}\left\{\left(r_{1}, \ldots, r_{k}\right) \in C_{k}: r_{i} \leq \varepsilon\right\}$ is a sum of bands with boundaries $x_{i}=0$ and $x_{i}=\varepsilon$ for $i=1, \ldots, k$. By $\mathcal{T}_{5}$ we denote the family of all tolerance relations of the form $\tau_{4}(\varepsilon)$.

5 . The tolerance relation $\tau_{5}$ is defined by a disjunction of atomic formulas

$$
\langle x, y\rangle \in \tau_{5}\left(\varepsilon_{1}, \ldots, \varepsilon_{k}\right) \Leftrightarrow \bigvee_{a_{2} \in A}\left[\delta_{a_{i}}(x, y) \leq \varepsilon_{i}\right],
$$

where $\varepsilon_{1, \ldots, \varepsilon_{k}}$ are non-negative real numbers. This relation is a generalization of the relation "min" (Figure 4a).

6. Our last example is a tolerance relation defined by

$$
\langle x, y\rangle \in \tau_{6}(w) \Leftrightarrow \prod_{a_{\imath} \in A} \delta_{a_{i}}(x, y) \leq w,
$$

where $w \in R^{+}$. The set $\overline{\tau_{6}(w)}$ is equal to $\left\{\left(r_{1}, \ldots, r_{k}\right) \in C_{k}: r_{1} \cdot \ldots \cdot r_{k} \leq w\right\}$. Hence it is a region in $C_{k}$ bounded by hyperboloid (Figure $4 \mathrm{~b}$ ). 


\section{Heuristics}

The time complexity of the searching problem for optimal tolerance relation parametrized by $k$ parameters for a set of $n$ objects is $O\left(n^{k}\right)$, because we have to test all possible values of parameter vector, where the number of possible values for one parameter is usually $O(n)$. This time is not feasible, when the dimension of the problem is large (the number of points $n$ and the dimension $k$ of the space are large ). We show, that the approximations of some tolerance relations can be constructed if its geometrical description is known. Below we present heuristics for two important tolerance relation classes.

\subsection{Searching for description conjunction}

The first example of tolerance relation classes is the descriptor conjunction $\tau\left(\varepsilon_{1}, \ldots, \varepsilon_{k}\right)$ (see (3)) having the following interpretation

$$
\overline{\tau\left(\varepsilon_{1, \ldots,}, \varepsilon_{k}\right)}=\left\{\left(r_{1}, \ldots, r_{k}\right) \in C^{k}: 0 \leq r_{i} \leq \varepsilon_{i} \text { for } i=1, \ldots, k\right\}
$$

One can see that for given $\varepsilon_{1, \ldots, \varepsilon_{k}}$, the set $(5)$ is included in the interval $I\left(\varepsilon_{1}, \ldots, \varepsilon_{k}\right)$ from $R^{k}$. Our goal is to search for parameters $\varepsilon_{1, \ldots}, \varepsilon_{k}$ such that the interval $I\left(\varepsilon_{1}, \ldots, \varepsilon_{k}\right)$ consists of "white" points only and, at the same time, as many as possible of them.

We start from the empty interval $I_{0}$ (with one null boundary, let $\varepsilon_{1}=0$ ). The idea of the algorithm is based on construction of a sequence of intervals by transforming any successive interval $I_{i}$ to a new interval $I_{i+1}$. Among generated intervals, we choose the best one. Transformation is performed by gradual augmenting the parameter $\varepsilon_{1}$, and by decreasing some of the remaining parameters so, that the interval $I_{i}\left(\varepsilon_{1}, \ldots, \varepsilon_{k}\right)$ is still consisting of while points only and including as many as possible points. The algorithm can be presented as follows:

Input: The set of labeled points from the space $R^{k}$.

Output: Parameters $\left\{\varepsilon_{1}, \ldots, \varepsilon_{k}\right\}$ of the sub-optimal interval.

1. Set $\varepsilon_{1}=0$ and $\varepsilon_{i}=\infty$ for $i=2, \ldots, k$.

2. Gradually augment the value of $\varepsilon_{1}$ to obtain a new interval $I\left(\varepsilon_{1}, \ldots, \varepsilon_{k}\right)$

3 . If the interval $I\left(\varepsilon_{1}, \ldots, \varepsilon_{k}\right)$ contains black points then decrease some of the remaining parameters to eliminate "black" points from interval $I\left(\varepsilon_{1}, \ldots, \varepsilon_{k}\right)$. We choose such parameters to optimize the number of "white" points belonging to the modified interval.

4. Repeat Step 2 until all possible values of $\varepsilon_{1}$ are checked. Return parameters $\varepsilon_{1}, \ldots, \varepsilon_{k}$ of the optimal interval among considered in Step 3.

The algorithm can be implemented in $O\left(n^{2} \cdot k\right)$ time by using sorted lists of possible values of considered parameters. 


\subsection{Searching for linear combination (hyperplane)}

Let us consider a linear combination tolerance relation $\tau\left(w_{1}, \ldots, w_{k}, w\right)$ ant its interpretation

$$
\overline{\tau\left(w_{1}, \ldots, w_{k}, w\right)}=\left\{\left(r_{1}, \ldots, r_{k}\right) \in C_{k}: \sum_{i=1}^{k} w_{i} \cdot r_{i}+w \leq 0\right\}
$$

For given parameters $w_{1}, \ldots, w_{k}, w$ the formula (6) describes the set of points with positive coordinates lying below the hyperplane $H: \sum_{i=1}^{k} w_{i} \cdot x_{i}+w=0$. This hyperplane is determined by $(k+1)$ parameters. Any hyperplane divides the space into two half-spaces. We say the hyperplane $H$ is satisfactory if the half-space below the hyperplane $H$ contains only "white" points. Our goal is to search for a satisfactory hyperplane $H$ with the (semi-)maximal number of "white" points below it.

The algorithm starts with randomly chosen hyperplane $H=\sum_{i=1}^{k} w_{i} \cdot x_{i}+w$. We generate a set of satisfactory hyperplanes starting from $H$. Among them we choose the best one. The satisfactory hyperplane can be computed on two stages. At first we rotate the hyperplane $H$ to obtain a new hyperplane (i.e. determines a new partition of point set). Then we translate it until the points below hyperplane are all "white". The hyperplane $H$ can be rotated by fixing $k$ parameters, for example $w, w_{1}, \ldots, w_{j-1}, w_{j+1}, \ldots, w_{k}$ and modifying only one parameter $w_{j}$. We are interested in a values of $w_{j}$ such that the modified hyperplane determines a new partition of set of objects. The idea is based on observation that a point $p=\left[x_{1}, x_{2}, \ldots, x_{k}\right] \in C^{k}$ is below $H$ iff $H(p) \leq 0$ i.e. $w_{j} \leq \frac{-\sum_{i \neq j} w_{i} \cdot x_{i}-w}{x_{j}}$. Let $w_{j}(p)=\frac{-\sum_{i \neq j} w_{i} \cdot x_{i}-w}{x_{j}}$ and

$$
S=\left\{w_{j}(p): p=\left[a_{1}^{\prime}(u), \ldots, a_{k}^{\prime}(u)\right] \text { for any } u \in U^{\prime}\right\}
$$

then any value $w_{j}$ chosen from $S$ determines a new hyperplane defining a new partition of the point set. The algorithm can be presented briefly as follows:

Input: The set of labeled points of the space $R^{k}$.

Output: Parameters $\left\{w, w_{1}, \ldots, w_{k}\right\}$ of the optimal satisfactory hyperplane.

1. $H\left(w, w_{1, \ldots,}, w_{k}\right):=$ randomly chosen hyperplane;

2. for (any $j=1 \ldots k$ )

\section{begin}

Construct the set $S$ defined in (7) and sort $S$ in increasing order;

for (any positive $v \in S$ )

begin

$w_{j}:=v$;

Translate $H\left(w, w_{1, \ldots,}, w_{k}\right)$ to a good position i.e. with all "white" points below it and calculate the number of those white points. The fitness of the hyperplane is equal to this number. end end 
3. Among good hyperplanes we choose a hyperplane with maximal fitness.

The algorithm repeats the Loop $2 O(k)$ times and every loop takes $O(k \cdot n)$ times. Therefore the complexity of proposed algorithm is $O\left(k^{2} \cdot n\right)$, where $n$ is a number of points and $k$ is the dimension of a space.

\section{Tolerance relation in classification problems}

\subsection{Clustering method}

Let $\mathbf{A}=(U, A)$ be an information system. Given a consistent tolerance relation $\tau$ defined on the universe $U$ we define its $\tau^{*}$ by $\tau^{*}=\bigcup_{n \geq 0} \tau^{n}$. The cluster $C$ can be defined as the object set such, that if $x, y \in C$ then $x \tau^{*} y$. The clusters of the universe $U$ can be constructed in a straightforward way

repeat

Choose $x \in U, C=[x]_{r *}$;

$U=U \backslash C$

until $U=\emptyset$

One can see that clusters determined by the algorithm are disjoint and they contain the objects with the same decision. We can use those clusters for classification of new cases in different ways. One example of classification strategy is presented below:

Step 1 : Every cluster $C_{i}$ is characterized by its center $\mathbf{c}_{i}$ and its mass $\mathbf{m}_{i}$ (Number of objects belonging to the cluster $C_{i}$ );

Step 2 : Define the distance function $d$;

Step 3 : For a new object $x$, the number $p_{i}(x)=\frac{m_{i}}{d\left(c_{i}, x\right)}$ is a gravitation power measure of the cluster $C_{i}$ influencing the new object $x$. The new case $x$ is classified to the cluster with the maximal gravitation power $p_{i}(x)$.

\subsection{Classification: Nearest Neighbours Method}

For a given tolerance $\tau$ and any object $x$ one can define the set of neighbours of $x$ in the tolerance sense. The set of neighbors of $x$ is defined gradually as follows:

$N N_{1}(x)=\{y: y \tau x\}$

$N N_{k}(x)=\left\{y: \bigvee_{z \in N N_{k-1}(x)} x \tau z \wedge z \tau y\right\}$

Having a set of neighbours of the object $x$, one can classify $x$ using different strategies, for example one can take a majority rule as the standard criterion. Classification process of new objects is presented below

Step 1 : Construct the set of neighbours $N N_{k}(x)$ of $x$ for some $k$. We choose the value $k$ in such way that the set $N N_{k}(x)$ contains no less then $M$ objects from training set. 
Step 2 : Use $M$ nearest neighbours of $x$ to vote for the decision on $x$. The object $x$ is classified to the decision class supported by the maximal number of objects from the $N N_{k}(x)$.

\section{Conclusion}

We have presented a new approach for extraction relational patterns in data. These patterns are described by tolerance relations extracted from data. The searching problem for optimal patterns can be transformed to some geometrical problems because almost all standard tolerance relations can be described by some regions in the space $R^{k}$. Hence one can extract tolerance relations from data by constructing approximation of corresponding regions. We have proposed some heuristic for the some important parametrized relation classes. We are working on the implementation of proposed methods.

Acknowledgement: This paper was supported by the State Committee for Scientific Research grant, KBN 8T11C01011.

\section{References}

1. Bezdek J.C., Chuah S., Leep D. Generalized k-Nearest Neighbour Rule, Fuzzy Sets and Systems, 1,8(3), 1986, pp.237-256.

2. Cover T., Hart P., Nearest Neighbour Pattern Classification, IEEE Trans. Inf. Theory, Vol.13, 1967, pp. 21-27.

3. Hu X., Cercone N., Rough Set Similarity Based Learning from Databases. Proc of The Fourth International Workshop on Rough Set, Fuzzy Set and Machine Discovery. August 20-21,1995 Montreal, Canada, pp.162-167.

4. Krawiec K., Stowiński R., Vanderpooten D. Construction of Rough Classifires Based on Application of a Similarity Relation. Proc. of The Fourth International Workshop on Rough Set, Fuzzy Set and Machine Discovery. November 6-8,1996, Tokio, Japan, pp.23-30.

5. Lin T.Y. Neighbourhood system and approximation in database and knowled base systems, Proc. of The Fourth International Symposium on Methodologies of Intelligent System, 1989.

6. Marcus S., Tolerance Rough Sets, Cech Topologies, Learning Process, Bull. of The Polish Academy of Technical Sciences, Vol. 42, No. 3, 1994, pp.471-487.

7. Nguyen S. H., Nguyen T. T., Skowron A., Synak P., Knowledge Discovery by Rough Set Methods, Proc. of The International Conference On Information Systems Analysis and Synthesis, July, 22-26, 1996, Orlando, USA, pp.26-33.

8. Nguyen S. H., Polkowski L., Skowron A., Synak P., Wróblewski J., Searching for Approximate Description of Decision Classes, Proc.of The Fourth International Workshop on Rough Sets, Fuzzy Sets, and Machine Discovery, November 6-8, 1996, Tokyo, Japan, pp.153-161.

9. Pawlak Z. Rough Classification, International Journal of Man-Machine Studies, 20, pp. 469-483, 1984.

10. Pawlak Z,, Rough Sets. Theoretical Aspects of Reasoning about Data, Kluwer Academic Publishers, Dordrecht, 1991. 
11. Polkowski L., Skowron A., Zytkow J., Tolerance Based Rough Sets, In: Soft Computing, T.Y.Lin, A.M. Wildberger (eds.), San Diego, Simulation Council, Inc., 1995, pp. $55-58$.

12. Stepaniuk J., Similarity Based Rough Sets and Learning, Proc. of The Fourth International Workshop on Rough Sets, Fuzzy Sets and Machine Discovery, November 6-8, 1996, Tokyo, Japan, pp.18-22.

13. Stepaniuk J., Krȩtowski M., Polkowski L., Skowron A., Data Reduction Based on Rough Set Theory, Proc. of The International Workshop on Statistics, Machine Learning and Knowledge Discovery in Databases, Crete, Greece, April 28-29,1995

14. Skowron A., Polkowski L., Komorowski J. (1996). Learning Tolerance Relation by Boolean Descrip tions: Automatic feature extraction from data tabes. Proc of The Fourth International Workshop on Rough Set, Fuzzy Set and Machine Discovery. November 6-8,1996, Tokio, Japan, pp.11-17.

15. Skowron A., Stepaniuk J., Tolerance Approximation Spaces. In Fundamenta Informaticae, August 1996, Vol. 27, Numer 2,3, pp.245-253.

16. Windham, M.P. Geometric Fuzzy Clustering Algorithms, Fuzzy Set and Systems 3 , pp.271-280, 1983.

17. Ziarko, W.P.(Ed.) Rough Set, Fuzzy Set and Knowledge Discovery, SpringerVerlag, London, 1994. 


\section{Appendix}

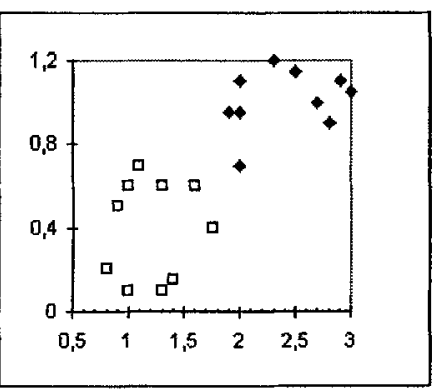

Fig. 1,a) The set of apples and pears

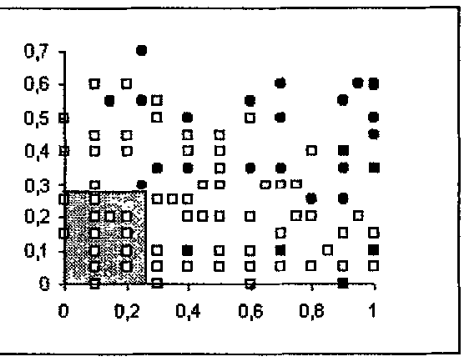

Fig. 2a) Tolerance relation $T_{1}$

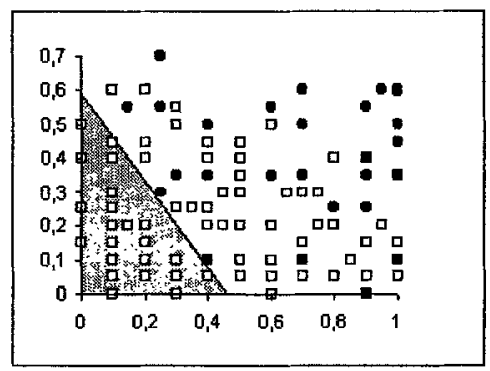

Fig. 3a) Tolerance relation $\mathcal{T}_{3}$

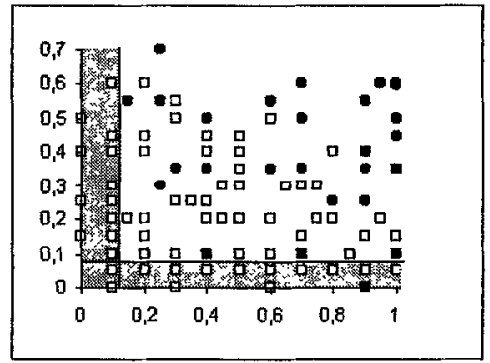

Fig. 4a) Tolerance relation $\mathcal{I}_{6}$

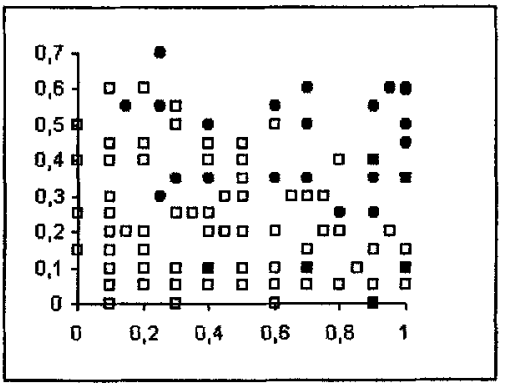

Fig. 1.b) The set of fruit pairs

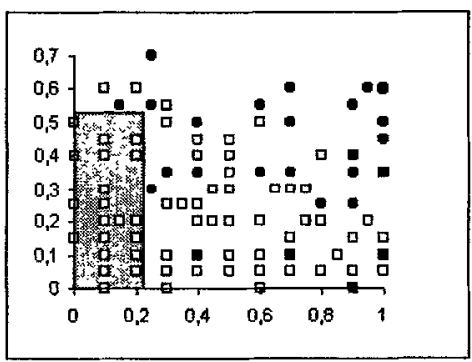

Fig. 2b) Tolerance relation $\mathcal{T}_{2}$

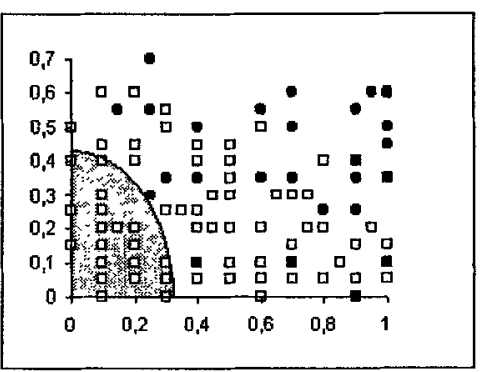

Fig. 3b) Tolerance relation $T_{4}$



Fig. 4b) Tolerance relation $T_{7}$ 